\title{
Misdiagnosis of seizures: insulinoma presenting as adult- onset seizure disorder
}

\author{
T D Graves*, S Gandhi*, S J M Smith, S M Sisodiya, G S Conway
}

J Neurol Neurosurg Psychiatry 2004;75:1091-1092. doi: 10.1136/jnnp.2004.029249

Once diagnosed with a refractory seizure disorder, patients often receive aggressive and escalating pharmacotherapy. However, a significant proportion of patients referred to neurologists do not have epilepsy. Toxic and metabolic causes of seizures should always be considered as they are potentially curable, and may be fatal if untreated. The following case report highlights the need for careful reassessment of all seizures that are atypical and refractory to medication.

\section{A} 44 year old woman was referred for management of refractory seizures. At birth she had been diagnosed with congenital toxoplasmosis. She had bilateral congenital cataracts and underwent a series of ophthalmological procedures, culminating in enucleation of the left eye at the age of 23, and was registered blind. Her developmental milestones were normal. She was otherwise well and had a healthy daughter. Her symptoms began at the age of 40 when she experienced monthly episodes of confusion and disorientation. Over the next 4 years she suffered from a mixture of "funny turns" with episodes lasting for 15 min when she would fidget, yawn repetitively, and be inaccessible. At other times she would collapse to the floor, her body would shake, and she might shout out sentences whilst collapsed. She was incontinent of urine in half of these attacks and was drowsy and confused for up to $3 \mathrm{~h}$ afterwards. Apart from a prosthetic left eye and right-sided blindness, neurological and general examination were unremarkable. She had been diagnosed with complex partial seizures and secondarily generalised seizures and commenced on antiepileptic medication. Despite escalating treatment, her seizure frequency and pattern had worsened significantly during the previous 2 years.

Her medications consisted of levetiracetam $1 \mathrm{~g}$ bd, lamotrigine $100 \mathrm{mg}$ mane and $125 \mathrm{mg}$ nocte, carbamazepine CR $700 \mathrm{mg}$ bd, and clobazam $10 \mathrm{mg}$ daily. Previous trials of sodium valproate and gabapentin were ineffective. A previous MRI brain scan demonstrated a few white matter lesions compatible with congenital toxoplasmosis. An EEG had shown an absence of normal cerebral rhythms over both hemispheres, with excess slowing in the left temporal region.

During the course of her admission the patient had a habitual "generalised seizure" whilst on ambulatory EEG and video recording. She was found lying on the floor rolling from side to side, shaking her head, and moaning. There were no tonic or clonic components. She was cold, sweaty, and intermittently confused but able to respond to questions. A finger prick test revealed a glucose level of $0.8 \mathrm{mmol} / \mathrm{l}$. The attack was terminated with $1 \mathrm{mg}$ intramuscular glucagon. EEG monitoring showed a gradual build up of bilateral anterior slow wave activity prior to the attack, increasing over $10 \mathrm{~min}$ and persisting for a further $30 \mathrm{~min}$, at which time a few sharp waves and spikes were seen anteriorly. The slow wave activity began to attenuate 17 min after glucagon was administered. Approximately 90 min after the clinical onset the EEG had returned to its baseline state and finger prick glucose was recorded at $2.8 \mathrm{mmol} / \mathrm{l}$. The EEG findings suggested a metabolic encephalopathy. The worsening and resolution of the slow wave changes directly mirrored blood glucose levels and the clinical state of the patient.

The patient was subsequently transferred to the Endocrine Unit. Her plasma glucose was maintained with a continuous infusion of 5\% dextrose. On further questioning it became apparent that these attacks tended to occur between 6 and 8 a.m. and 4 and 6 p.m. They were relieved by food or sugary drinks to the extent that she had gained $25 \mathrm{~kg}$ in weight. Fasting plasma glucose was $2.4 \mathrm{mmol} / \mathrm{l}$ (normal range 3.96.0) with a concomitant insulin level of $186.1 \mathrm{mU} / \mathrm{l}$ (normal range 5-20) and C-peptide level of $1322 \mathrm{pmol} / \mathrm{l}$ (normal range 260-650). A urine sulphonylurea screen was negative. Computerised tomography of the abdomen showed a $2.3 \mathrm{~cm}$ hypervascular mass in the head of the pancreas and a $1.6 \mathrm{~cm}$ mass in the body. At operation further small tumours were found, necessitating Whipple's procedure. Histological examination revealed at least 12 insulinomas with no features of malignancy. The patient made a good recovery and is now seen as an outpatient for the control of her post-pancreatectomy diabetes mellitus. Antiepileptic drugs have been discontinued with no recurrence of her attacks.

\section{DISCUSSION}

We report a case of multiple insulinomas presenting as an adult-onset refractory seizure disorder. The time from onset of symptoms to diagnosis was 4.5 years. The atypical features of her attacks, which were inconsistent with complex partial and generalised seizures, combined with her poor response to treatment prompted an inpatient assessment. It was following direct observation during a habitual attack, aided by ambulatory EEG recording, that the diagnosis of neuroglycopenia could be made. This case highlights the importance of considering hypoglycaemia in atypical neurological or psychiatric episodes and emphasises the role of inpatient evaluation of refractory epilepsy.

Insulinomas are the commonest hormone-secreting tumour of the gastrointestinal tract; the incidence is 0.5-1 cases/million/year, although this is likely to be an underestimate. There is a female preponderance with onset usually in middle age; tumours are usually solitary, benign, and sporadic, although 5-10\% occur in patients with MEN-1, 10\% are metastatic, and a further $10 \%$ are multiple but behave as benign tumours. The only diagnostic feature of malignancy is the presence or subsequent development of metastases. Insulinomas are found throughout the pancreas and are small (usually $10-20 \mathrm{~mm}$ ). Diagnosis relies on inappropriate insulin secretion $(>30 \mathrm{pmol} / \mathrm{l})$ in the presence of hypoglycaemia $(<2.2 \mathrm{mmol} / \mathrm{l})$ and subsequent tumour localisation. ${ }^{1}$ 


\begin{tabular}{|ll|}
\hline \multicolumn{2}{|l|}{ Table 1 Symptoms of hypoglycaemia } \\
\hline Neuroglycopenia & Autonomic \\
\hline Confusion & Sweating \\
Lethargy, stupor, or coma & Tremor \\
Bizarre behaviour & Palpitations \\
Personality change & Anxiety \\
Amnesia & Weakness \\
Decreased motor activity & Visual disturbance \\
Transient neurological deficit & \\
Gradual decline in cognition & \\
\hline Adapted from Jenner. & \\
\hline
\end{tabular}

Presentation is usually insidious with neuroglycopenia (table 1) and fasting hypoglycaemia. As seen in our case, this may lead to a delay in diagnosis as other neuropsychiatric diagnoses may be considered first. ${ }^{1}$ In a retrospective study of 59 patients with histologically confirmed islet cell adenomas, the interval between onset of symptoms and diagnosis ranged from 1 month to 30 years, with a median of 24 months. A significant proportion (39\%) were originally diagnosed with a seizure disorder. Interestingly, all patients had symptoms of neuroglycopenia and three quarters reported relief of symptoms with ingestion of food. ${ }^{2}$ In a prospective study involving 25 patients presenting to a neurology outpatient department with "funny turns", patients with variable and atypical attacks were admitted to an assessment unit for imaging, EEG, and a $72 \mathrm{~h}$ fast with repeat EEG recording. Two cases $(8 \%)$ demonstrated significant hypoglycaemia with inappropriately high insulin levels and were subsequently found to have benign insulinsecreting adenomas. ${ }^{3}$ Despite these data, only four cases of insulinoma presenting with seizures have been reported in Western literature..$^{4-7}$ Uncontrolled hypoglycaemia, as well as being potentially fatal, can have serious neurological sequelae, such as cognitive impairment and peripheral neuropathy. ${ }^{4}$

Neuroglycopenia should be considered in all patients with "funny turns", seizures, and other neuropsychiatric symptoms, especially if they do not conform to diagnostic criteria or respond to standard treatment. Taking a full history (including relationship of attacks to food, non-stereotyped or atypical attacks, and poor response to antiepileptic treatment) and clinical suspicion are the key to making a diagnosis of insulinoma. Once suspected, confirming the diagnosis with a $72 \mathrm{~h}$ fast is relatively simple. Benign insulinsecreting adenomas are a potentially curable cause of seizures.

\section{CONTRIBUTORS}

Sonia Gandhi and Sanjay Sisodiya looked after the patient at Chalfont, Shelagh Smith interpreted the EEGs, and Tracey Graves and Gerald Conway looked after the patient at Middlesex Hospital. Tracey Graves and Sonia Gandhi wrote the first draft.

\section{Authors' affiliations}

T D Graves, G S Conway, Department of Endocrinology, Middlesex Hospital, UCLH NHS Trust, London, W1A 8NN, UK

S Gandhi, S J M Smith, S M Sisodiya, National Society for Epilepsy, Chalfont St Peter, Buckinghamshire, SL9 ORJ, UK

Competing interests: none declared

*These authors contributed equally to this paper

Correspondence to: Sanjay Sisodiya, National Society for Epilepsy, Chalfont St Peter, Buckinghamshire, SL9 ORJ, UK; sisodya@ion.ucl.ac.uk

Received 29 September 2003

In revised form 29 September 2003

Accepted 13 January 2004

\section{REFERENCES}

1 Marks V, Teale JD. Tumours producing hypoglycaemia. Endocr Relat Cancer 1998;5:111-29.

2 Dizon AM, Kowalyk S, Hoogwerf BJ. Neuroglycopenic and other symptoms in patients with insulinomas. Am J Med 1999;106(3):307-10.

3 Harrington MG, McGeorge A, Ballantyne JP, et al. A prospective survey for insulinomas in a neurology department. Lancet 1983;14:1094-5,

4 Crimmins D, Marks J, McLeod JG, et al. Fits, confusion and peripheral neuropathy in a young woman. Aust New Zealand J Med 1990;20:623-8.

5 Hui ACF, Ma RCW, Kay R, et al. Seizure recurrence in temporal lobe epilepsy. Int J Clin Pract 2002;56(1):63-4.

6 Bazil CW, Pack A. Insulinoma presenting as seizure disorder. Neurology 2001;56:817-8.

7 Jenner GH. A seizure and odd behaviour in a 79-year-old man. Postgrad Med J 1995;71(842):761-3. 